\title{
Design, construction and quality control of resistive-Micromegas anode boards for the ATLAS experiment
}

\author{
F. Kuger ${ }^{1,2, a}$, P. lengo ${ }^{1}$, and on behalf of the ATLAS Muon Group \\ ${ }^{1}$ CERN, Geneva $(\mathrm{CH})$ \\ ${ }^{2}$ Bayerische Julius Maximilians Universität Würzburg (DE)
}

\begin{abstract}
For the upcoming upgrade of the forward muon stations of the ATLAS detector, $1280 \mathrm{~m}^{2}$ of Micromegas chambers have to be constructed. The industrialization of anode board production is an essential precondition. Design and construction methods of these boards have been optimized towards mass production. In parallel quality control procedures have been developed and established. The first set of large size Micromegas anode boards has finally been produced in industries and demonstrates the feasibility of the project on full-scale.
\end{abstract}

\section{Introduction}

The New Small Wheels (NSWs), an upgrade of the innermost stations of the ATLAS endcap muon detectors, will be installed during the LHC long shutdown in 2018-19 [1]. The 128 modules of Micromegas (MM) detectors [2], each one consisting of eight layers, to be installed on the two 10 -meter-diameter wheels will comprise a total active detector area of $1280 \mathrm{~m}^{2}$.

The Micromegas anode boards, representing the key component of the detectors, are manufactured in industries, making the NSW Micromegas the first MPGD for a large experiment with its key component industrially produced. A total of 2048 anode boards, divided in 32 different types, each $0.5 \mathrm{~m}$ wide and up to $2.2 \mathrm{~m}$ long, are required for the construction of both NSWs.

This article summarizes the design of the NSW MM anode boards (Sec.2) and describes the steps of their production in industries with the corresponding quality requirements (Sec.3). The quality control (QC) scheme to be applied during this production is presented (Sec.4) and the results from a test production for NSW MM Module-0 are reported (Sec.5). Finally the status of large size Micromegas anode board production in industries is summarized (Sec.6).

\section{Anode board design}

The NSW Micromegas anode boards are a stacked structure of several layers, as shown in Fig. 1.

The copper readout structure comprises 1023 readout strips of $300 \mu \mathrm{m}$ width, covering the active area of the detector. The routing of the readout strips accounts for the positioning of assembly holes without strip cuts or interruptions (Fig.2d). Half of the strips are routed on the left or

\footnotetext{
a e-mail: fabian.kuger@cern.ch
}

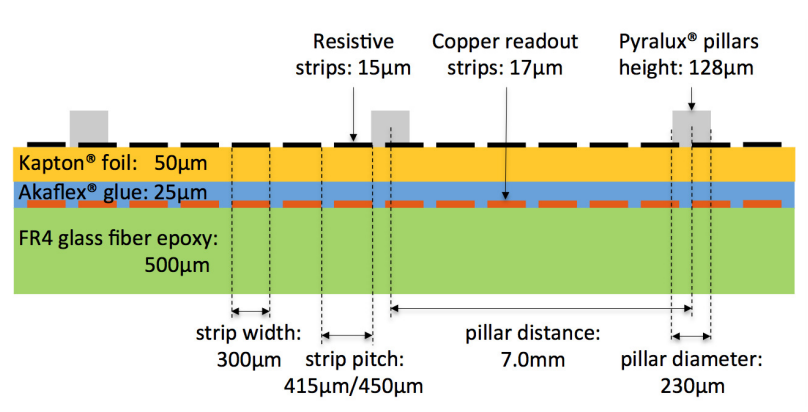

Figure 1: Schematic of the NSW Micromegas anode board layers and dimensions of its main structures (different strip pitch for small / large NSW sector PCBs). Not to scale.

right to connector pads (Fig.2a) where the signal is transmitted to the front-end electronics via an elastomeric connector, avoiding soldering on the boards. Additionally the outer borders include, among other features, crossmark targets for precision holes (Fig.2c), Rasnik Masks [4] for positioning and dimensions control (Fig.2b), alignment targets and a HV supply line including space for $\mathrm{HV}$ filters per side (Fig.2e).

A layer of strips printed with resistive ink on an insulating Kapton ${ }^{\circledR}$ foil follows the pattern of the readout strips. Interconnections between those lines are placed every $10 \mathrm{~mm}$ in an alternate configuration to achieve a homogeneous surface resistivity. The resistive layer provides protection for the readout electronics and spark suppression improving the high-rate capability of the detector [3]. High voltage is applied to the resistive layer from the copper pattern via a hole in the Kapton ${ }^{\circledR}$ foil. A line of silver polymer ink ensures a low impedance connection to the end of each strip (Fig.2e). The resistive strips are inter- 
rupted in their middle (Fig.2d) to achieve a finer granularity in the high voltage distribution.

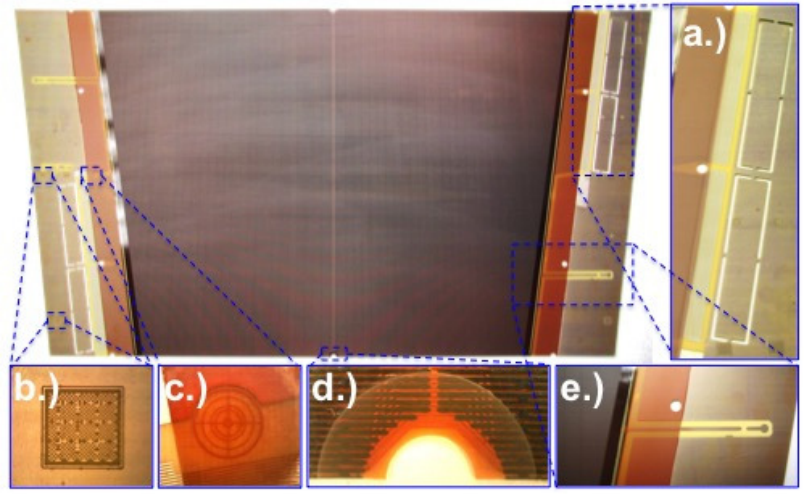

Figure 2: A NSW MM anode board with magnification of a.) the connector pad region including holes and the cutout for electronic cooling; b.) a Rasnik Mask on various positions; c.) the precision target; d.) the routing of strips around a hole and resistive layer interruption in the board center; e.) HV supply point on the copper pattern and part of the silver conduct line.

Cylindrical pillars with a diameter of $230 \mu \mathrm{m}$ and a thickness of $128 \mu \mathrm{m}$ are arranged in a triangular array $7 \mathrm{~mm}$ aside all over the detector area. They define, once the electrically grounded metallic mesh is applied on top, the amplification gap.

The boards are left-right symmetric to allow for installation of the same board types in a back-to-back doublet configuration. While on one doublet (eta) the strips will run perpendicular to the precision coordinate (and parallel to each other), the strips are inclined by $\pm 1.5^{\circ}$ on the second doublet (stereo). This allows for the reconstruction of the precision coordinate with a spatial resolution $\mathrm{O}(100 \mu \mathrm{m})$ in both configurations and of the second coordinate with a reduced resolution $\mathrm{O}(2-3 \mathrm{~mm})$ on a stereo doublet [5].

\section{Production process and quality requirements}

Although the design of the anode boards allows standardized industrial processes during production, the combination of these techniques, which are partially adopted from different branches of industry, and the large size of the boards result in a challenging production process. Additionally the physics performance and the mechanical requirements set tight quality constrains and acceptance criteria on the anode board.

The full production process is visualized in Fig. 3 and each technique is shortly described in the following, pointing out the major quality requirements for the corresponding production step.

\subsection{Copper pattern creation}

The readout pattern is etched into the copper layer $(17 \mu \mathrm{m})$ on temperature stabilized glass fiber epoxy (FR4, $0.5 \mathrm{~mm}$

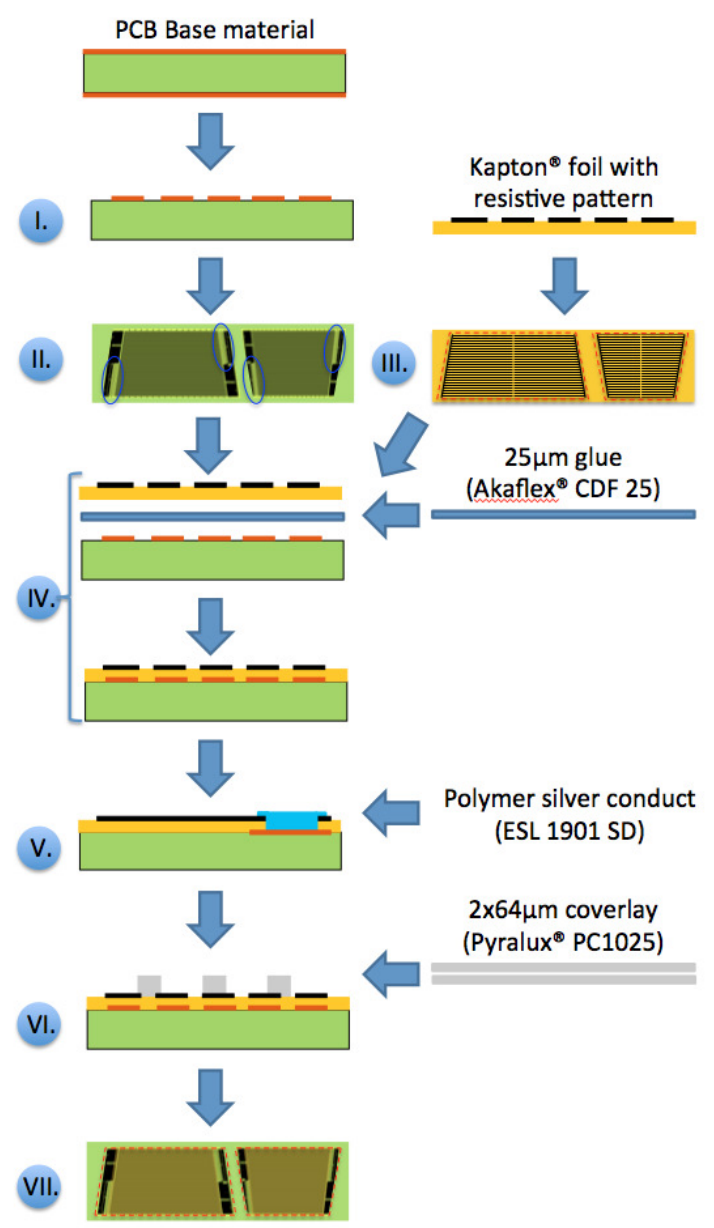

Figure 3: Process chart of the anode board construction, showing base materials and the different production steps: I. Copper pattern creation; II. Connector pad plating; III. Kapton ${ }^{\circledR}$ foil cutting; IV. Kapton ${ }^{\circledR}$-PCB gluing; V. Silver conduct application; VI. Pillar pattern creation; VII. Cutting and drilling.

$\pm 50 \mu \mathrm{m})$ via classical photo-lithographic processes [6] in a temperature and humidity controlled environment.

The required absolute accuracy is $\pm 30 \mu \mathrm{m}$ for the precision coordinate direction (short side) to guarantee accurate positioning of the readout strips in the detector, a precondition to achieve the required spacial resolution. On the long side $\pm 100 \mu \mathrm{m} / \mathrm{m}$ accuracy are required. The local copper pattern inaccuracy, e.g. deviation in line thickness, is tolerated to $\pm 20 \mu \mathrm{m}$. Shorts between two lines can be repaired and are only tolerated on $<0.1 \%$ level, while up to $1 \%$ of non-repairable strip interruptions are accepted if not on neighboring strips.

\subsection{Selective plating on the connector pads}

To ensure a perfect conductivity between the connector pads and the elastomeric connectors, the copper has to be covered with a noble metal like $\mathrm{Au}, \mathrm{Ag}$ or Pd using either electroless-/immersion-plating with a mediating Ni layer [7] or electroplating processes [6]. 
A complete and homogeneous coverage of the pads is required, where the layer thickness depends on the choice of the plating but ranges for all in $\mathrm{O}(1 \mu \mathrm{m})$.

\subsection{Kapton ${ }^{\circledR}$ foil preparation and cutting}

The resistive pattern is applied on a Kapton ${ }^{\circledR}$ foil $(50 \mu \mathrm{m})$ via screen printing using a resistive ink (ESL D-RS 12115) [8], resulting in strips with a thickness of $\approx 15 \mu \mathrm{m}$. This corresponds to an initial resistivity of $0.3-0.4 \mathrm{M} \Omega / \square$, resulting in the target resistivity of $\approx 0.8 \mathrm{M} \Omega / \square$, after an increase during the subsequent production steps.

The resistive layers are industrially produced in Japan and delivered to the PCB contractor. They have to be cut to size with moderate accuracy of $\pm 1 \mathrm{~mm}$ and cleaned thoroughly.

\subsection{Gluing of Kapton ${ }^{\circledR}$ foil on PCB}

The Kapton ${ }^{\circledR}$ foil is glued on the readout side of the PCB with a $25 \mu \mathrm{m}$ thick Akaflex ${ }^{\circledR}$ glue layer under high pressure of $5-7 \mathrm{~kg} / \mathrm{cm}^{2}$ at $170^{\circ} \mathrm{C}$. An alignment accuracy of $<0.5 \mathrm{~mm}$ is required between the two layers.

Good cleanliness is essential to avoid enclosures between layers and dents on the surface. To reduce inhomogeneity in pressure, leading to air inclusion between the copper strips, additional layers of conformity material (Pacoflex ${ }^{\circledR}(0.27 \mathrm{~mm})$ or Pacotherm ${ }^{\circledR}(0.89 \mathrm{~mm})$ ) are added to the stack before pressing and removed afterwards. A homogeneous, enclosure-free gluing with a flat surface is demanded, since small bumps on the surface of the anode could create discharges in the detector. The change in resistivity during the pressing has to be monitored.

\subsection{Application of polymer silver conduct}

The conductive line, connecting the HV input pad and the end of the resistive strips, is applied either manually or by screen printing using ESL 1901 SD silver conduct.

A coarse positioning of $\pm 1 \mathrm{~mm}$, flatness of the conduct line and a low impedance have to be guaranteed.

\subsection{Coverlay and pillar pattern creation}

After lamination with a double layer of $64 \mu \mathrm{m}$ Pyralux ${ }^{\circledR}$ PC1025, the pillar pattern is transferred via a photolithographic process [6] with a positioning accuracy of $\pm 1 \mathrm{~mm}$ referring to the copper pattern.

The essential requirement is an (almost) complete pillar pattern, where missing pillars are only allowed if non consecutive and less than $0.1 \%$ in total. Neighboring missing pillars create the risk of discharges in the detector due to larger sagging of the metallic mesh between the supporting pillars. A maximum of ten extra structures and other defects on the coverlay are allowed if minor in size $\left(<1 \times 1 \mathrm{~mm}^{2}\right)$. To obtain a homogeneous gain in the detector, a constant height of the pillars on $<5 \mu \mathrm{m}$ level is required.

\subsection{Board cutting and drilling of assembly holes}

The finalized anode boards are milled parallel to the readout strips to size with a CNC machine on $<100 \mu$ m precision. The tight margin is necessary to avoid overlapping anode boards when joining them along the strips to a full detector plane.

Non-precise assembly holes are drilled with the same accuracy requirement along the outside borders of the detector plane.

\section{Quality control and quality assurance}

Given the complexity of the process a single quality control after finalization of the production proved to be insufficient to achieve a satisfying yield, limit material losses and trace systematic production-related errors. In close feedback with the involved industries we established a multistep quality control plan, shown in Fig.4.

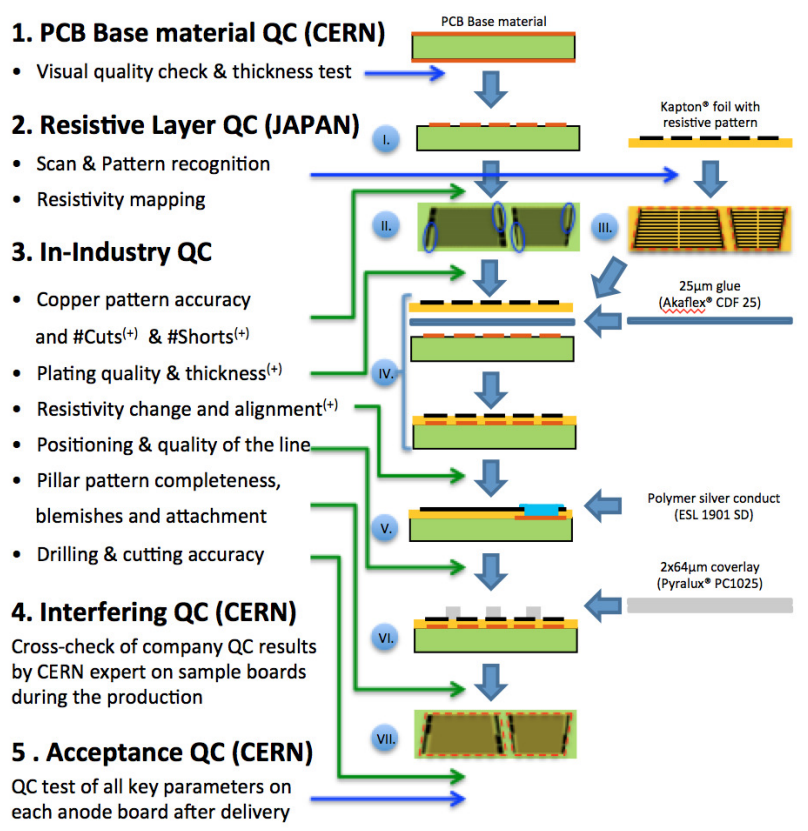

Figure 4: Visualization of the 5 stages of QC/QA during NSW MM anode board production, including the key parameters to be tested after each production step.

The relevant parameters of each production step are tested before further processing of the specific part. This minimizes material waste since unqualified boards are rejected immediately and only qualified components are further used.

Accordingly, the two supplies under responsibility of our collaboration, the PCB base material and the Kapton ${ }^{\circledR}$ foils with resistive pattern, undergo a QC procedure before being shipped to industries. This additionally enables us to track the change of material attributes during the production, like the resistivity increase, and distinguish between production faults and damages occurred during processing in industries.

Performing QC between each step of production requires involvement from industries and their operators. As 
shown in Fig.4 each key parameter of the different production steps will be tested immediately, where on some items (marked with ${ }^{(+)}$) specified QC results shall be reported while on others compliance statements are considered sufficient. Facilitating these controls by e.g. integrated QC markers was one objective during the design phase. Two examples are rings on the copper pattern to verify drilling accuracy by simple visual control (Fig.5 top) and direct readable alignment targets (Fig. 5 bottom).
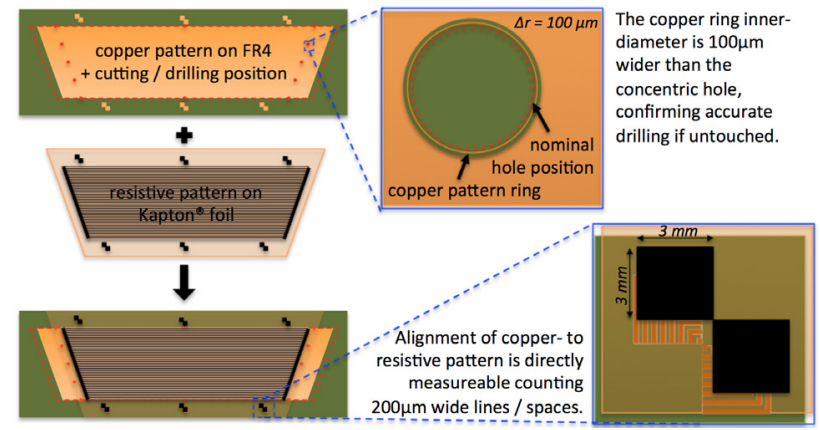

Figure 5: Left: Stacking scheme of FR4 with copper pattern, Kapton ${ }^{\circledR}$ with resistive layer and milling nominal positions. Right: Two kind of design-integrated QC markers.

Cross-checking these QC results obtained in industries by CERN personnel is foreseen during the full production. While a tight follow up during the production start-up will reduce the time for optimization of procedures and machine tuning, regular inspection during the running production are planned to maintain QC/QA standards and accordingly maximize the production yield.

Each finalized board will undergo an full quality control after delivery to CERN. Additionally to checking the key parameters, methods requiring dedicated tooling, not available in industries, can be applied at CERN. Examples are time efficient dimensional measurements $(\mathrm{O}(5 \mu \mathrm{m})$ accuracy) on a specially developed contact-CCD-table, $\mathrm{O}(1 \mu \mathrm{m})$-precise pillar height mappings and full surface resistivity measurements. Given the large quantity of boards to be tested time-efficiency, simplicity and repeatability of the methods where the main objectives during the development of the procedures and the tooling.

\section{QC/QA results of first anode boards}

The first anode boards to be used for the construction of NSW Micromegas full-scale prototype detectors (Module0 ) have been produced in the summer of 2015 by two companies. The boards have undergone a control of quality at CERN to verify the compliance with the requirements. While some assumed critical parameters, like the homogeneity of the pillar height, were fulfilled on most of the boards, several common issues have been discovered in both productions:

- Deviations in the dimensions of the copper pattern

- Bubbles or dust between the Kapton ${ }^{\circledR}$ and the PCB

- Missing and weakly attached pillars
- Inaccurate and unclean edge cutting and hole drilling

These issues have been addressed with regular feedback and visits to the company's premises, leading to subsequent deliveries of higher quality boards. Although the overall yield of acceptable boards during Module- 0 production is suffering from these start-up problems, the latest delivery included anode boards fully compliant with all requirement, demonstrating the feasibility of the production scheme and requirements in industrial processes.

\section{Conclusion}

The development work carried out at CERN to push the Micromegas technology to a new frontier is now coming to an end. The production of the first read-out boards for the upgrade of the ATLAS muon system demonstrates in full-scale the feasibility of the project.

A tender process to assign the production of the 2048 boards needed for the NSW project is ongoing and the mass production will start in the first half of 2016 and span over about 18 months.

PCB quality requirements are being finalized and quality control procedures in industries are established. The preparations for a final QC at CERN are in the last step of tooling preparation. Readiness of the PCB quality control procedures before the start of the series production is foreseeable.

\section{References}

[1] ATLAS Collaboration: ATLAS New Small Wheel Technical Design Report, CERN-LHCC-2013-006, ATLAS-TDR-020-2013, Geneva (2013).

[2] Y. Giomataris, et al: MICROMEGAS: a highgranularity position-sensitive gaseous detector for high particle-flux environments, Nucl. Instrum. Meth. A 376 (1996), 29-35.

[3] T. Alexopoulos, et al: A spark-resistant bulkmicromegas chamber for high-rate applications, Nucl. Instrum. Meth. A 640 (2011), 110-118.

[4] S. Aefsky, et al: The Optical Alignment System of the ATLAS Muon Spectrometer Endcaps, Journal of Instrumentation, Volume 3 (2008).

[5] M. Bianco, et al: Construction of two large-size four-plane micromegas detectors, arXiv:1511.03884 [physics.ins-det] (2015).

[6] S. Franssila: Introduction to Microfabrication, John Wiley \& Sons, Ltd, 2nd Edition, Chichester (2010).

[7] J. Sudagar, J. Lian, W. Sha: Electroless nickel, alloy, composite and nano coatings - A critical review, Journal of Alloys and Compounds, Volume 571 (2013), 183204.

[8] N. Serra: Epoxy-graphite Resistive Composites: Formulation, Characterization and Applications, EPFL Thesis No. 5346, Lausanne (2012). (doi:10.5075/epflthesis-5346) 